\title{
Case Report: Emphysematous Pyelonephritis Triggers Diabetic Ketoacidosis in A 60 Years Old Patient
}

\author{
Gerasimos Kapellos $^{1^{*}}$, Natalia Fourla ${ }^{2^{*}}$ and Vlassios Polychronopoulos ${ }^{3}$ \\ ${ }^{1}$ Department of Internal Medicine, Sismanoglio General Hospital, Marousi, Greece \\ ${ }^{2}$ Department of Medicine, Resident Doctor, Athens, Greece \\ 3Internal Medicine Department, Pulmonary Clinic, Hygeia Hospital, Athens, Greece
}

"Corresponding author: Natalia Fourla, MD, Department of Medicine, Resident Doctor, Athens, Greece, E-mail: natalyfourla@gmail.com; Gerasimos Kapellos, MD, PhD, Department of Internal Medicine, Sismanoglio General Hospital, Marousi, Greece, Tel: 0030/6976805348; E-mail: g.kapellos@hotmail.com Received date: November 11, 2018; Accepted date: November 26, 2018; Published date: November 29, 2018

Copyright: (c) 2018 Kapellos G, et al. This is an open-access article distributed under the terms of the Creative Commons Attribution License, which permits unrestricted use, distribution, and reproduction in any medium, provided the original author and source are credited.

\begin{abstract}
The report describes the case of a 60 years old female patient admitted in our emergency department with main complaints of abdominal pain, malaise, fever and oliguria the last three days. The primary blood samples investigations revealed leukocytosis, acute renal function impairment and increased serum glucose. The patient is known with diabetes type II for the last fifteen years, has been in treatment with metformin and basic insulin therapy but the disease remains not well controlled. Further laboratory investigation and physical examination were consistent with the diagnosis of diabetic ketoacidosis. The urine examination showed pyuria leading to the diagnosis of a urinary tract infection. The abdominal XRAY was without abnormal findings but in the ultrasound and abdominal CT, a few renal parenchymatic lesions have been observed, more clearly seen on the CT. The presence of air within the tissue was consistent with a very rare and life-threatening infection, known as emphysematous pyelonephritis. The patient was put on the necessary treatment.
\end{abstract}

Keywords: Emphysematous; Pyelonephritis; Infection; Diabetes; Complication; Diabetic ketoacidosis

\section{Introduction}

Pyelonephritis is a quite common infection in young and older adult women with a mean age of 58 years old [1]. Patients usually receive the appropriate treatment without complications. In diabetic patients, however, especially in women, there is a very rare type of pyelonephritis which may be found. Emphysematous pyelonephritis is a rare and severe renal infection associated with presence of gas bubbles within the tissue of the kidney. In the onset of the disease, patients have a mild symptomatology which can progress to renal failure [2]. The most common agent responsible for this infection is $\mathrm{E}$. Coli which is found in the urine cultures in almost $70 \%$ of cases, others being Klebsiella, Proteus, Pseudomonas, Clostridium species and sometimes polymicrobial infections. The diagnosis of this rare disease is mainly confirmed by abdominal CT [3].

In patients with diabetes, any infection may be the stimulus leading to diabetic ketoacidosis. In our patient, the acidic blood $\mathrm{pH}$ and the presence of ketones were activated by inflammation of renal parenchyma in a non-well controlled diabetes type 2. Diabetic ketoacidosis refers to very low insulin level unable to control high level of glucose so that the fat burning mechanism is altered. The described syndrome is an acute metabolic disorder characterized by hyperglycemia, ketones bodies and metabolic acidosis. Its prognosis is not that good and still remains a disorder with high mortality rate [4]. The most important factors which may lead to diabetic ketoacidosis are infections or inappropriate diabetes treatment. The confirmation of the disease is made by the laboratory tests such as high blood glucose, ketone bodies and metabolic acidosis in arterial blood gases in association with abdominal CT scans. All these findings were present in our patient's investigations. The treatment of diabetic ketoacidosis has mainly the goal to hydrate the patient and decrease the level of glucose in the blood, in order to control the acid-base balance [4].

\section{Case Study}

A 60 years old lady was admitted in the emergency department of our hospital with intense abdominal pain in the right flank, fever and oliguria. The vital signs were as follows: normal arterial blood pressure, increased body temperature $\left(38.2^{\circ} \mathrm{C}\right)$. She was looking very ill and she had a slight anemic appearance. On auscultation, she had normal S1, S2 and normal breathing sounds. On palpation, she presented tenderness in the abdominal region mainly in the right flunk with radiation to the back and positive Giordano sign. Her past medical history revealed diabetes type 2 and no other chronic diseases nor medication was reported.

The laboratory tests revealed; leukocytosis 20.000/Ml, HTC 37\% and hemoglobin $12 \mathrm{~g} / \mathrm{dl}$. The leukocytosis was attributed to the concurrent infection which needed to be treated with antibiotic therapy. Abnormal laboratory findings included glucose $580 \mathrm{mg} / \mathrm{dl}$, urea $120 \mathrm{mg} / \mathrm{dl}$, creatinine $2 \mathrm{mg} / \mathrm{dl}$, potassium level $5.3 \mathrm{mmol} / \mathrm{l}$, sodium level $139 \mathrm{mmol} / \mathrm{l}$, liver enzymes AST and ALT $77 \mathrm{U} / \mathrm{L}$. Arterial blood gases examination revealed pH 7, p CO2 15 mmHg and HCO3 3 $\mathrm{mmol} / \mathrm{L}$. These findings confirmed the diagnosis of metabolic acidosis. A blood culture was performed and revealed E. coli as specific agent, same as in the urine culture. Urine had glucose $(++++)$, ketone $(+++$ + ), and pyuria more than 200 cells, some bloods cells and small range proteinuria.

Taken account all the above clinical and laboratory findings, the diagnosis of diabetic ketoacidosis was confirmed and the presence of concomitant urine infection was also confirmed. In order to establish 
the level of infection, ultrasound and abdominal CT were performed and revealed enlarged right kidney along with damaged parenchyma and a rare finding of air captured inside the tissue. The above finding was compatible with emphysematous pyelonephritis.

The treatment strategy was mainly to correct ketoacidosis and infection. The patient received IV fluids and insulin for DKA and compatible antibiotic treatment (Figure 1).

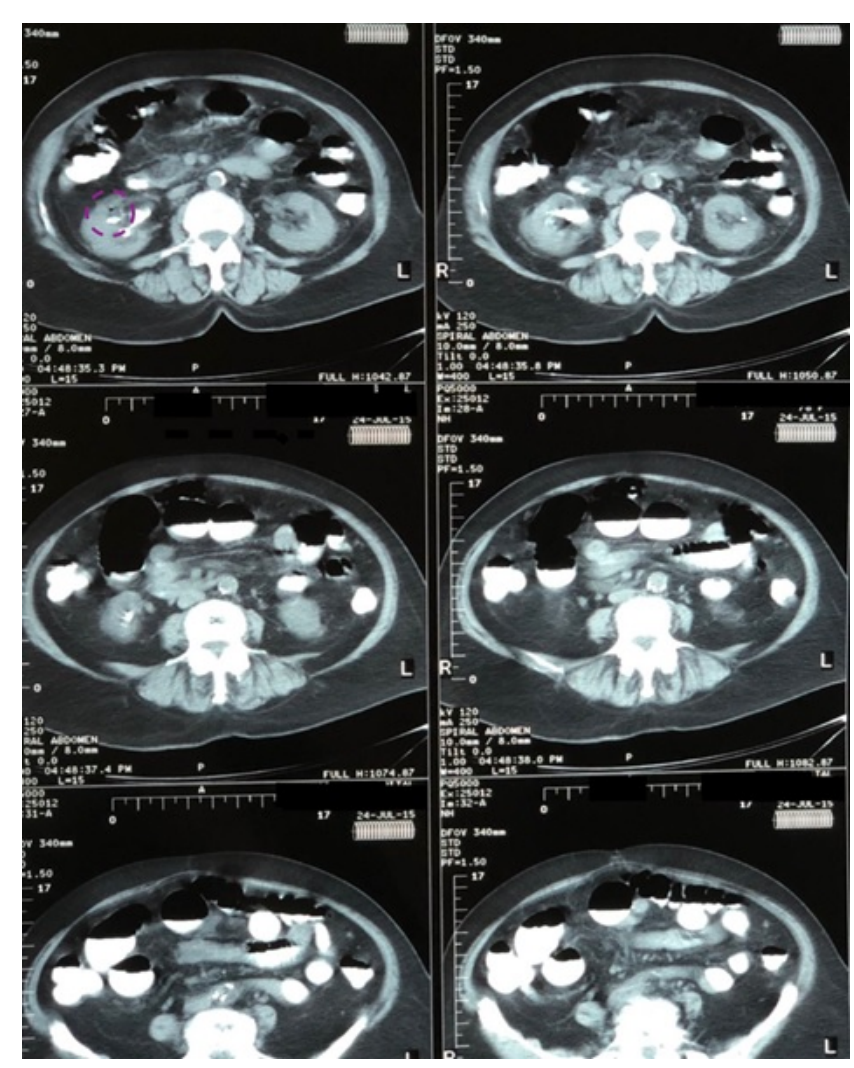

Figure 1: CT of 60 years old patient is class 2 according to the classification of emphysematous pyelonephritis based on the CT radiological findings [5].

\section{Discussion}

Emphysematous pyelonephritis is a very rare and severe infection of the kidney. It's associated with presence of gas in the parenchyma with or without extension in the perinephric tissue. The most commonly found cause of the disease is infection by Escherichia Coli followed by Klebsiella. EPN is considered a rare renal infection, however with increasing prevalence of diabetes, metabolic syndrome and increasing use of computed tomograms (CT), increasing number of cases are diagnosed now [2].

The treatment is based on administration of antibiotics but in many cases, nephrectomy cannot be avoided. Despite the treatment, mortality remains high at a percentage of $21 \%[6,7]$.

In our case, the diagnosis was confirmed by CT, which showed an impressive image, presence of air within the right kidney without abscess or necrosis of the tissue. Clinically EPN presents with nonspecific features of upper urinary tract infection including fever, flank pain, nausea, vomiting, altered sensorium, shock, acute renal failure and disseminated intravascular coagulation [2].

According to the classification of stages, based on radiological findings, our patient was in class 2 . This stage has to do only with limited presence of air without expansion in the perinephric tissue [5].

It's known, that in patients with diabetes, infections are very common and may lead to a severe complication such as diabetic ketoacidosis. This case is quite interesting because it's very uncommon, for this suppurative infection, to be associated with diabetic ketoacidosis. Diabetic ketoacidosis (DKA) is very severe condition which needs immediate treatment plan and follow up, because could be fatal. Our patient, diagnosed with DKA, was immediately hydrated and received intravenous insulin therapy. The management was successful.

In most of the cases, surgical intervention with antibiotics is the appropriate treatment of emphysematous pyelonephritis, but in our case, despite the severe comorbidities the patient received only conservative therapy and no surgical intervention was required. This is why our case study was reported [8].

\section{Conclusion}

Diabetes is known to be associated with an increased risk of infection. In our case, emphysematous pyelonephritis was the leading cause of a complication of diabetes, known as diabetic ketoacidosis. These cases need to be treated immediately, in order to correct the metabolic acidosis, eradicate the infection and avoid nephrectomy. Our case combines two different severe conditions, which if they left untreated, may be fatal for the patient.

\section{References}

1. Chung VY, Tai CK, Fan CW, Tang CN (2014) Severe acute pyelonephritis: a review of clinical outcome and risk factors for mortality. Hong Kong Med J 20: 285-289.

2. Sokhal AK, Kumar M, Purkait B, Jhanwar A, Singh K, et al. (2017) Emphysematous pyelonephritis: changing trend of clinical spectrum, pathogenesis, management and outcome. Turk J Urol 43: 202-209.

3. Hiremath R, Mahesh, Padala KP, Swamy K, Pailoor A (2015) A rare case of pneumoureter: emphysematous pyelitis versus emphysematous pyelonephritis. J Clin Diagn Res 9: TD03-TD05.

4. Fayfman M, Pasquel FJ, Umpierrez GE (2017) Management of hyperglycemic crises: diabetic ketoacidosis and hyperglycemic hyperosmolar state. Med Clin North Am 101: 587-606.

5. Huang JJ, Tseng CC (2000) Emphysematous pyelonephritis: Clinicoradiological classification, management, prognosis, and pathogenesis. Arch Intern Med 160: 797-805.

6. Somani BK, Nabi G, Thorpe P, Hussey J, Cook J et al. (2008) Is percutaneous drain- age the new gold standard in the management of emphyse- matous pyelonephritis? Evidence from a systematic review. J Urol 179(5): 1844-1849.

7. Sharma PH, Sharma R, Vijay MK, Tiwari P, Goel A, et al. (2013) Emphysematous pyelonephritis: our experience with conservative management in 14 cases. Urol Ann 5: 157-162.

8. Deoraj S, Zakharious F, Nasim A, Missouris C (2018) Emphysematous pyelonephritis: outcomes of conservative management and literature review. BMJ Case Rep pii: bcr-2018-225931. 